\title{
Cooperative Diversity Using Optimal Relay Power Control for Different Fading Channels
}

DOI:

10.1109/WCNC.2017.7925523

\section{Document Version}

Accepted author manuscript

Link to publication record in Manchester Research Explorer

\section{Citation for published version (APA):}

Alfitouri, A., \& Hamdi, K. (2017). Cooperative Diversity Using Optimal Relay Power Control for Different Fading Channels. In 2017 IEEE Wireless Communications and Networking Conference IEEE.

https://doi.org/10.1109/WCNC.2017.7925523

\section{Published in:}

2017 IEEE Wireless Communications and Networking Conference

\section{Citing this paper}

Please note that where the full-text provided on Manchester Research Explorer is the Author Accepted Manuscript or Proof version this may differ from the final Published version. If citing, it is advised that you check and use the publisher's definitive version.

\section{General rights}

Copyright and moral rights for the publications made accessible in the Research Explorer are retained by the authors and/or other copyright owners and it is a condition of accessing publications that users recognise and abide by the legal requirements associated with these rights.

\section{Takedown policy}

If you believe that this document breaches copyright please refer to the University of Manchester's Takedown Procedures [http://man.ac.uk/04Y6Bo] or contact uml.scholarlycommunications@manchester.ac.uk providing relevant details, so we can investigate your claim.

\section{OPEN ACCESS}




\title{
Cooperative Diversity Using Optimal Relay Power Control for Different Fading Channels
}

\author{
Abdurrahman Alfitouri, and Khairi Ashour Hamdi \\ School of Electrical and Electronic Engineering, \\ The University of Manchester, Manchester, M13 9PL, UK \\ Emails: \{Abdurrahman.alfitouri, k.hamdi\}@manchester.ac.uk
}

\begin{abstract}
In this paper, we study the performance of a wireless communication system, where an arbitrary number of relays cooperate to enhance the signal between source and destination. The source and destination are hidden from each other, and there is no direct link between them. Optimal Relay Power Control (ORPC) is introduced as an effective scheme to achieve cooperative diversity, where there is a trade-off between complexity and system performance. This paper develops a new and more accurate model for the ORPC scheme and takes into account the effects of fading and thermal noise at both the relays and destination. This leads to the derivation of new analytical expressions for the overall spectral efficiency which can be used to estimate the throughput of the ORPC in Rayleigh and Nakagami$m$ fading channels, and to study the impact of different system parameters on efficiency. The accuracy of the new mathematical results is confirmed by Monte Carlo simulation. The results of this paper can be used to estimate the aggregate throughput of a dual-hop system employing the ORPC protocols.

Index Terms-Cooperative diversity, amplify-and-forward, $\mathrm{Op}$ timal Relay Power Control.
\end{abstract}

\section{INTRODUCTION}

Cooperative diversity network technology, see [1], [2], is known to be an effective means of high data-rate coverage and a means of coping with the fading of wireless channels, both of which are necessary for future cellular and ad-hoc wireless communication systems. Various cooperation diversity protocols have been proposed; these include amplify-and-forward (AF) and decode-and-forward (DF) [3], [4]. Multiple antennas have been considered as cooperative diversity protocols which uses increased diversity to combat channel fading [5]. When each antenna at the transmitter sends the same signal information to another antenna at the receiver, multiple copies of the data symbols can arrive at the receiver node and this can lead to an improvement in system performance.

Maximum ratio transmission (MRT) is considered as the best technique to combine multiple relays signals and to achieve maximum data rate. However, the complexity of the MRT is greater than for any other technique, and each relay in the network needs to know every channel between the source, relays, and destination. On the other hand, the best relay selection (BRS) scheme is cooperation scheme that attracted considerable interests because it can achieve full diversity with fewer synchronization or feedback requirements. However, the achievable data rate for BRS is less than the MRT, because only one relay carries the data between the source and destination. In this regard, optimal relay power control (ORPC) is introduced as an effective means of achieving cooperative diversity. ORPC is a trade-off between complexity and system performance. The performance of an ORPC cooperative diversity system is significantly better than for BRS, but lower than for MRT. On the other hand, ORPC has less complexity at both the relays and the destination since the relay needs to know only its own channels [6], [7].

The analyses of cooperative protocol in home power line communication is reported in [8]. The performance of cooperative diversity using equal gain combinations over Nakagami$\mathrm{m}$ fading channels is discussed in [9]. The single and multiple relay selection schemes and their achievable diversity orders are reported in [10]. The end-to-end performance of a twohop system with non-regenerative relays over flat Rayleighfading channels is proposed in [11]. For a Rayleigh-fading environment, analysis of the average symbol error rate for a distributed spatial diversity wireless system with amplifying relays is presented in [12].

In this paper, we analyse the performance of a random number of active relays applying ORPC scheme to enhance the signal between the source and its destination, where there is no direct link between them. This model experience two different channel fading namely, Rayleigh and Nakagami-m fading channels. The analysis taking into account (in addition to the random number of cooperative relays) the effects of fading and thermal noise at both the relays and the destination. To the best of the authors' knowledge, system where the number of the cooperative relays is treated as random have not been considered in the literature but is related to many practical scenarios. However, this assumption makes the proposed model more practical than previous models. The proposed formula reveals the implication of source activity on the overall spectral efficiency (SE) of the system.

We derive explicit expressions for the SE of the ORPC model, which can be used to estimate the throughput of this system, and to study the impact of the different system parameters on efficiency, where the new results are expressed in terms of the weights and abscissas of a Laguerre orthogonal polynomial. The SE of ORPC for different models of channel fading, such as Rayleigh and Nakagami-m fading, has not previously been addressed in the literature.

The SNR in this model is a ratio of a large number of random variables. However, the direct approach to finding the overall SE may require at least $(3 M)$-fold numerical 
integrations, which is difficult to obtain in general. As result, we investigate the use of a simple and useful Lemma 1 to evaluate this averaging. Moreover, the numerator of the SNR is not a liner sum, since it is the square of the sum of multiple random variables, which cannot be directly solved. Therefore, we use corollary 2 to simplify this equation, where the result includes a simple mathematical equation that can be easily evaluated. We compare our scheme with some popular schemes, such as the BRS, and MRT. Furthermore, simulation results are provided to validate our analysis.

The remainder of this paper is organized as follows. Section II introduces the system model. The SE analysis for ORPC is explained in section III. The SE analysis in Rayleigh fading channels, and the exact-form are presented in Section IV. In Section V, the SE analysis for Nakagami-m fading channels, and the exact-form are presented. Results are presented and discussed in Section VI. Finally, concluding remarks are given in Section VII.

\section{System Model}

The system model under consideration has one source, one destination, and each of these nodes is equipped with a single antenna, which can be used for both transmission and reception. There are $M$ number of relays distributed in the service area around the source and the destination. The source and destination are hidden from each other with no direct link between them. The communication between the source and the destination is divided into two phases. During the first phase, the source sends its signal to the relays simultaneously. In the second phase, a random number of relays forward the amplified signal to the destination. The channel from the source to the $i^{t h}$ relay is denoted as $h_{i}$ and the channel from the $i^{\text {th }}$ relay to the destination as $g_{i}$. All channels are assumed to be subject to complex Gaussian fading with zero mean and unit variance, e.g., $h_{i}, g_{i} \backsim C N(0,1)$. All the additive white Gaussian noise (AWGN) terms related to all links $(S \rightarrow$ Relays $\rightarrow D$ ), are assumed to have zero mean and variance $\left(\sigma_{r i}^{2}\right.$, and $\sigma_{d}^{2}$, respectively.). Without loss of generality, in the first phase, the received signal at the $i^{t h}$ relay is given by

$$
y_{i}=h_{i} l_{i}^{-\frac{\alpha}{2}} x+n_{i}
$$

where $x$ is the transmitted symbol with unit power, $h_{i}$ is the complex channel gain between the source and relay $i, l_{i}$ is the distance between the source and relay $i, \alpha$ is the path loss exponent, and $n_{i}$ is AWGN at the $i^{t h}$ relay. During the second phase, a random number of relays amplifies $y_{i}$ by multiplying it with $w_{i}$, which is the weight of relay $i$, and broadcasts it to the destination. The received composite signal at the destination in the $\mathrm{BC}$ phase is given by

$$
y_{D}=\sum_{i=1}^{M} g_{i} \delta_{i} w_{i} r_{i}^{-\frac{\alpha}{2}}\left(h_{i} l_{i}^{-\frac{\alpha}{2}} x+n_{i}\right)+n_{d}
$$

where $g_{i}$ is the complex channel gain between the destination and relay $i, r_{i}$ is the distance between the relay $i$ and destination, $n_{d}$ is AWGN at the destination, and $\delta_{i}$ is random variable representing the status of each relay (cooperating or not), where

$$
\delta_{i}=\left\{\begin{array}{cc}
1 & \text { if relayicooperates } \\
0 & \text { otherwise }
\end{array}\right.
$$

At each relay, the channel state information (CSI) is available, which mean that the relay knows the cooperating channels between $(S \rightarrow$ Relays $\rightarrow D)$. Therefore, the weight of the relay $i$ can be expressed as

$$
w_{i}=\frac{1}{\sqrt{M}} \frac{h_{i}^{*}}{\left|h_{i}\right|} \frac{g_{i}^{*}}{\left|g_{i}\right|}
$$

Therefore, the received signal at the destination can be written as

$$
\begin{aligned}
y_{D} & =\frac{1}{\sqrt{M}} \sum_{i=1}^{M} \\
& \left(\delta_{i}\left|h_{i}\right|\left|g_{i}\right| r_{i}^{-\frac{\alpha}{2}} l_{i}^{-\frac{\alpha}{2}} x+\delta_{i}\left|g_{i}\right| \frac{h_{i}^{*}}{\left|h_{i}\right|} r_{i}^{-\frac{\alpha}{2}} n_{i}\right)+n_{d} .
\end{aligned}
$$

Thus, the SNR at the destination node is given by

$$
\mathrm{SNR}=\frac{\left(\sum_{i=1}^{M} \delta_{i}\left|h_{i}\right| l_{i}^{-\frac{\alpha}{2}}\left|g_{i}\right| r_{i}^{-\frac{\alpha}{2}}\right)^{2}}{\sum_{i=1}^{M} \delta_{i}\left|g_{i}\right|^{2} r_{i}^{-\alpha} \sigma_{r i}^{2}+M \sigma_{d}^{2}}
$$

\section{SPECTRAL EFFICIENCY ANALYSis}

In communication systems, the SE describes the data rate that can be sent over a specific bandwidth. In this section, we derive the achievable SE of the two phase relay system, which can be expressed as

$$
\Re=\frac{1}{2} \mathrm{E}\left[\log _{2}(1+\mathrm{SNR})\right]
$$

The achievable SE of the two phase relay system with EGT scheme can be written as

$$
\Re=\frac{1}{2} \mathrm{E}\left\{\log _{2}\left(1+\frac{\left(\sum_{i=1}^{M} \delta_{i}\left|h_{i}\right| l_{i}^{-\frac{\alpha}{2}}\left|g_{i}\right| r_{i}^{-\frac{\alpha}{2}}\right)^{2}}{\sum_{i=1}^{M} \delta_{i}\left|g_{i}\right|^{2} r_{i}^{-\alpha} \sigma_{r i}^{2}+M \sigma_{d}^{2}}\right)\right\} .
$$

Form Equation (8), we can see the effect of the random status of each relay on the system performance, where, when $\delta_{i}=0$, the SE equal zero since $\log \left(1+\frac{0}{0+M \sigma_{d}^{2}}\right)=0$, which means no relay cooperates to enhance the signal between the source and the destination, which result the SE being zero. The expectation in (7) is with respect to random variable SNR, which is the ratio of a large number of random variables, and it is difficult to derive a closed-form expression for its distribution. The overall SE of our proposed model can be found from (8), where we need to find the expectation of this equation with respect to the following set of $(3 M)$ random variables: $\mathbf{h}=\left\{h_{1}, h_{2}, \ldots, h_{M}\right\}, \mathbf{g}=\left\{g_{1}, g_{2}, \ldots, g_{M}\right\}$, and $\delta=\left\{\delta_{1}, \delta_{2}, \ldots, \delta_{M}\right\}$. The direct approach to compute this expectation is difficult to obtain in general, as it may require the computation of a ( $3 M)$-fold convolution integral. As result, 
in this paper we use the useful Lemma 1 to perform the averaging required in (8).

Lemma 1. [13, Eq. (5)] Let $x_{1}, \ldots, x_{N}, y_{1}, \ldots, y_{M}$ be arbitrary random variables $>0$. Then

$$
\begin{aligned}
\ln \left(1+\frac{\sum_{n=1}^{N} x_{n}}{\sum_{m=1}^{M} y_{m}+1}\right)= \\
\quad \int_{0}^{\infty} \frac{1}{z}\left(1-e^{-z \sum_{n=1}^{N} x_{n}}\right) e^{-z\left(\sum_{m=1}^{M} y_{m}+1\right)} d z .
\end{aligned}
$$

Thus, we can rearrange (8) to obtain the exact analytical expression for the SE as follows:

$$
\begin{aligned}
\Re=\frac{1}{2} \mathrm{E}\left[\left(\log _{2} e\right)\right. & \int_{0}^{\infty} \frac{1}{z}\left(1-e^{-z\left(\sum_{i=1}^{M} \delta_{i}\left|h_{i}\right|\left|g_{i}\right| r_{i}^{-\frac{\alpha}{2}} l_{i}^{-\frac{\alpha}{2}}\right)^{2}}\right) \\
& \left.\times e^{-z \sum_{i=1}^{M} \delta_{i}\left|g_{i}\right|^{2} r_{i}^{-\alpha} \sigma_{r i}^{2}} e^{-z M \sigma_{d}^{2}} d z\right] .
\end{aligned}
$$

The expectation in (10) for the term $e^{-z\left(\sum_{i=1}^{M} \delta_{i}\left|h_{i}\right|\left|g_{i}\right| r_{i}^{-\frac{\alpha}{2}} l_{i}^{-\frac{\alpha}{2}}\right)^{2}}$ is not easy to obtain, where the square in the exponent includes all the summation. We use corollary 2, which make the random variables appear only in a liner combination at the exponent, to simplify the evaluation of the required average.

Corollary 2. [14, Eq. (6)] the well-known identity

$$
\frac{1}{\sqrt{\pi}} \int_{-\infty}^{\infty} e^{-\omega^{2}} d \omega=1
$$

This is equivalent to $1=\frac{1}{\sqrt{\pi}} \int_{-\infty}^{\infty} e^{-(\omega+j u)^{2}} d \omega$, which implies $e^{-z u^{2}}=\frac{1}{\sqrt{\pi}} \int_{-\infty}^{\infty} e^{-\omega^{2}} e^{-j 2 \omega \sqrt{z} u} d \omega$. Because the left hand side is real, it follows that the right hand side should also be real. Thus $e^{-z u^{2}}=\frac{1}{\sqrt{\pi}} \int_{-\infty}^{\infty} e^{-\omega^{2}} \operatorname{Re}\left(e^{-j 2 \omega \sqrt{z} u}\right) d \omega$. According to [15], this means (10) can be written as

$$
\begin{array}{r}
\Re=\frac{1}{2}\left(\log _{2} e\right) \int_{0}^{\infty} \frac{e^{-z M \sigma_{d}^{2}}}{z}\left(\prod_{i=1}^{M} \mathrm{E}\left[e^{-z \sigma_{r i}^{2} \delta_{i}\left|g_{i}\right|^{2} r_{i}^{-\alpha}}\right]-\frac{1}{\sqrt{\pi}}\right. \\
\times \int_{-\infty}^{\infty} \operatorname{Re}\left(\prod_{i=1}^{M} \mathrm{E}\left[e^{\left.-\delta_{i}\left(z \sigma_{r i}^{2}\left|g_{i}\right|^{2} r_{i}^{-\alpha}+j 2 \omega \sqrt{z}\left|h_{i}\right|\left|g_{i}\right| r_{i}^{-\frac{\alpha}{2}} l_{i}^{-\frac{\alpha}{2}}\right)\right]}\right]\right) \\
\left.\times e^{-\omega^{2}} d \omega\right) d z
\end{array}
$$

This can be expressed as

$$
\Re=\frac{1}{2}\left(\log _{2} \mathrm{e}\right) \int_{0}^{\infty} \frac{e^{-z M \sigma_{d}^{2}}}{z}\left(\mathcal{M}_{u}(z)-\mathcal{M}_{v}(z)\right) d z
$$

where $\mathcal{M}_{v}(z)=\frac{1}{\sqrt{\pi}} \int_{-\infty}^{\infty} e^{-\omega^{2}} \operatorname{Re} \Phi(z \omega) d \omega$, and $\Phi(z \omega)=$ $\mathrm{E}\left[e^{-\delta_{i}\left(z \sigma_{r i}^{2}\left|g_{i}\right|^{2} r_{i}^{-\alpha}+j 2 \omega \sqrt{z}\left|h_{i}\right|\left|g_{i}\right| r_{i}^{-\frac{\alpha}{2}} l_{i}^{-\frac{\alpha}{2}}\right)}\right]$ is the character- istic function $(\mathrm{CHF})$ of the sum, and $\mathcal{M}_{u}(z)=\prod_{i=1}^{M} \mathrm{E}\left[e^{-z u}\right]$ is the MGF of $u$, where $u=\sigma_{r i}^{2} \delta_{i}\left|g_{i}\right|^{2} r_{i}^{-\alpha}$.

\section{RAYLEIGH FADING CHANNELS}

In the first scenario, all channels are assumed to be subject to independent non identical distributed complex Gaussian fading, with zero mean and unit variance, e.g., $h_{i}, g_{i} \backsim C N(0,1)$, where $i=\{1,2, \ldots, M\}$. As a result, the magnitude of channels, $h_{i}$ and $g_{i}$, follows a Rayleigh distribution. Therefore, the distribution of power channel gain, $\left|g_{i}\right|^{2}$ become exponential distribution.

\section{A. The $M G F$ of $u$}

The $\mathcal{M}_{u}(z)$ can be simplified to

$$
\begin{aligned}
\mathcal{M}_{u}(z)=\prod_{i=1}^{M} \mathrm{E}\left[e^{-z \sigma_{r i}^{2} \delta_{i}\left|g_{i}\right|^{2} r_{i}^{-\alpha} \mid \delta_{i}}\right] & \\
& =\prod_{i=1}^{M}\left(\frac{1}{1+z \sigma_{r i}^{2} \delta_{i} r_{i}^{-\alpha}}\right) .
\end{aligned}
$$

The term $\delta_{i}$ stands for the random status for relay $i$. In this system, we represent $\delta_{i}$ by independent Bernoulli random variable, where the probability, $\operatorname{Pr}\left(\delta_{i}=1\right)=\rho_{i}$ and $\operatorname{Pr}\left(\delta_{i}=0\right)=1-\rho_{i}$. Therefore, $\mathcal{M}_{u}(z)$ can be expressed as

$$
\mathcal{M}_{u}(z)=\prod_{i=1}^{M}\left(1+\rho_{i}-\frac{\rho_{i}}{1+z \sigma_{r i}^{2} r_{i}^{-\alpha}}\right)
$$

where $\rho_{i}$ is the probability that the relay $i$ is cooperating, and takes a value in the range $0 \leq \rho_{i} \leq 1$.

\section{B. The $M G F$ of $v$}

The equation for $\mathcal{M}_{v}(z)$ can be simplified and written as

$$
\begin{aligned}
& \mathcal{M}_{v}(z)=\prod_{i=1}^{M}\left(1+\rho_{i}-\rho_{i}\right. \\
& \left.\left(\frac{1}{\sqrt{\pi}} \int_{-\infty}^{\infty} e^{-\omega^{2}} \operatorname{Re}\left(\mathrm{E}\left[e^{-\left(a_{i}\left|g_{i}\right|^{2}+\omega b_{i}\left|h_{i}\right|\left|g_{i}\right|\right)}\right]\right) d \omega\right)\right)
\end{aligned}
$$

where $a=z \sigma_{r i}^{2} r_{i}^{-\alpha}$, and $b_{i}=j 2 \sqrt{z} r_{i}^{-\frac{\alpha}{2}} l_{i}^{-\frac{\alpha}{2}}$. The expectation in (16) is with respect to $h_{i}$, and $g_{i}$. Therefore, (16) can be simplify as (17), which shown at the top of the next page. Equation (17) gives the MGF of $v$ at distances $r$, and $l$, which can be obtained by substituting the values of $r$ and $l$ in $\mathcal{M}_{v}(z)$. It is worth mentioning that (17) can be expressed in terms of the weights and abscissas of a Laguerre orthogonal polynomial

$$
\begin{aligned}
& \mathcal{M}_{v}(z)=\prod_{i=1}^{M}\left\{1+\rho_{i}-\frac{\rho_{i}}{\sqrt{\pi}} \sum_{e=1}^{E} \Psi_{e}\right. \\
&\left.\operatorname{Re}\left(\frac{1}{\left(1+2 a-b^{2} \lambda_{e}^{2}\right)}-\frac{b \lambda_{e} \tan ^{-1}\left(\frac{\sqrt{1+2 a-b^{2} \lambda_{e}^{2}}}{b \lambda_{e}}\right)}{\left(1+2 a-b^{2} \lambda_{e}^{2}\right)^{3 / 2}}\right)\right\} \\
&+R_{E}
\end{aligned}
$$




$$
\mathcal{M}_{v}(z)=\prod_{i=1}^{M}\left\{1+\rho_{i}-\frac{\rho_{i}}{\sqrt{\pi}} \int_{-\infty}^{\infty} e^{-\omega^{2}} \operatorname{Re}\left(\frac{1}{\left(1+2 a_{i}-b_{i}^{2} \omega^{2}\right)}-\frac{b_{i} \omega \tan ^{-1}\left(\frac{\sqrt{1+2 a_{i}-b_{i}^{2} \omega^{2}}}{b_{i} \omega}\right)}{\left(1+2 a_{i}-b_{i}^{2} \omega^{2}\right)^{3 / 2}}\right) d \omega\right\}
$$

where $\lambda_{e}$ and $\Psi_{e}$ are the sample points and the weights factors of the Laguerre polynomial, respectively, tabulated in [16, Eq (25.4.46)]. For $E \geq 15$, the remainder $R_{E}$ is sufficiently small as to be ignored. Substituting (14) and (18) into (13) gives the final expression of overall SE.

\section{NAKAGAMI-M FADING CHANNELS}

In the second scenario, the magnitude of the complex channel gains, $h_{i}$ and $g_{i}$, is characterised by Nakagami-m fading channels, with two parameters: a shape parameter $m$, and a parameter, $\Omega$, controlling spread. The distribution of the power channel gain, $\left|g_{i}\right|^{2}$ is a gamma distribution. The $\mathrm{SE}$ in case of Nakagami-m channels is given by the unified expression (13). However, we need to evaluate $M_{u}(z)$ and $M_{v}(z)$ for the Nakagami-m model.

\section{A. The MGF of $u$}

The $\mathcal{M}_{u}(z)$ can be simplified to

$$
\begin{aligned}
\mathcal{M}_{u}(z)=\prod_{i=1}^{M} \mathrm{E}\left[e^{-z \sigma_{r i}^{2} \delta_{i}\left|g_{i}\right|^{2} r_{i}^{-\alpha} \mid \delta_{i}}\right] \\
=\prod_{i=1}^{M}\left(\frac{1}{1+\frac{z}{m} \sigma_{r i}^{2} \delta_{i} r_{i}^{-\alpha}}\right)^{m} .
\end{aligned}
$$

The last expectation in (19) is with respect to the random variable $\delta_{i}$. Therefore, $\mathcal{M}_{u}(z)$ can be expressed as

$$
\mathcal{M}_{u}(z)=\prod_{i=1}^{M}\left(1-\rho_{i}+\frac{\rho_{i}}{1+\frac{z}{m} \sigma_{r i}^{2} r_{i}^{-\alpha}}\right)^{m}
$$

\section{B. The MGF of $v$}

The equation for $\mathcal{M}_{v}(z)$ can be simplified and written as

$$
\begin{aligned}
& \mathcal{M}_{v}(z)=\prod_{i=1}^{M}\left(1-\rho_{i}+\rho_{i}\left(\frac{1}{\sqrt{\pi}} \int_{-\infty}^{\infty} e^{-\omega^{2}}\right.\right. \\
& \left.\left.\operatorname{Re}\left(\mathrm{E}\left[e^{-\left(z \sigma_{r i}^{2}\left|g_{i}\right|^{2} r_{i}^{-\alpha}+j 2 \omega \sqrt{z}\left|h_{i}\right|\left|g_{i}\right| r_{i}^{-\frac{\alpha}{2}} l_{i}^{-\frac{\alpha}{2}}\right)}\right]\right) d \omega\right)\right)
\end{aligned}
$$

The expectation in (21) with respect to $h_{i}$, and $g_{i}$. Therefore, $\mathcal{M}_{v}(z)$ can be simplify as (22), where it expressed in terms of the weights and abscissas of a Laguerre orthogonal polynomial, which shown at the top of next page, where $\vartheta_{c}$ and $\varphi_{c}$ are the sample points and the weights factors of the Laguerre polynomial, respectively, tabulated in [16, Eq. (25.4.46)]. For $C \geq 15$, the remainder $R_{C}$ is sufficiently small as to be ignored. Substituting (14) and (22) into (13) gives the final expression of overall SE. Equation (13) can also be expressed in terms of the weights and abscissas of a Laguerre orthogonal polynomial

$$
\Re=\frac{1}{2}\left(\log _{2} \mathrm{e}\right) \sum_{n=1}^{\mathrm{N}} \frac{\xi_{n}}{\beta_{n}}\left(\mathcal{M}_{u}\left(\mu_{n}\right)-\mathcal{M}_{v}\left(\mu_{n}\right)\right)+R_{N}
$$

where $\mu_{n}=\frac{\beta_{n}}{M \sigma_{d}^{2}}, \beta_{n}$ and $\xi_{n}$ are the sample points and the weights factors of the Laguerre polynomial, respectively, tabulated in [16, Eq. (25.4.45)]. For $N \geq 15$, the remainder $R_{N}$ is sufficiently small as to be ignored; therefore, (23) provides an efficient numerical evaluation method for the required $\mathrm{SE}$.

\section{NumERICAL AND Simulation RESUlts}

In this section, the SE achieved by the ORPC scheme is evaluated using Monte-Carlo simulation, and compared to the derived asymptotic results. The complex channel gains are modelled as Rayleigh and Nakagami-m distributions. Different graphical plots of SE are presented below, corresponding to various numbers of relays and relay statuses. We compare cooperative diversity networks which apply different schemes at the relays and the destination nodes for the special case, where all relays are active and $\delta=1$. For simplicity we assume $\rho_{1}=\ldots=\rho_{10}=\rho$, and $\sigma_{r 1}^{2}=\ldots=\sigma_{r 10}^{2}=\sigma_{r}^{2}$. The path loss exponent $(\alpha=3)$, shape index $(m=5)$, and $l_{i}=r_{i}=0.1$ $\mathrm{km}$.

\section{A. Rayleigh Fading Channel}

Fig. 1, shows the SE (in bits/s/Hz) of three different schemes as function of the number of relays, $M$, when $\sigma_{r}^{2}=\sigma_{d}^{2}=$ 1 watt $/ \mathrm{Hz}$. We can see that the theoretical ORPC curve is an excellent match to the simulated ORPC performance. We also see that an increase in the number of cooperating relay nodes (M) beneficially enhances the SE performance, and the achieved diversity order for the different schemes. In addition, it is evident that the performance of the ORPC cooperative diversity system is significantly better than the BRS, but not as good as the MRT. However, as explained above, the MRT is a much more complex system because of the need to know the channels of every relay; and the ORPC has less complexity at both the relays and the destination since each relay needs to know only its own channels.

In Fig. 2, the SE of ORPC is plotted as function of the number of relays between the source and destination that can communicate with both the source and destination, for different values of relay status $\rho$, when $\sigma_{r}^{2}=\sigma_{d}^{2}=1 \mathrm{watt} / \mathrm{Hz}$. We also see that an increase in the number of cooperating relay nodes (M) has a beneficial impact, enhancing the SE performance. From this figure, it is clear that the SE increases as the number of relays increases. In general, better performance is achieved the greater the value of $\rho$; this is because the number of sources of useful signal increase as $\rho$ increases, which leads to a better SNR value. 


$$
\begin{aligned}
& \mathcal{M}_{v}(z)=\prod_{i=1}^{M}[1+\rho_{i}-\frac{\rho_{i}}{\sqrt{\pi}} \sum_{c=1}^{C} \varphi_{c} \operatorname{Re}\left(( \frac { m ^ { m } l _ { i } ^ { \frac { - \alpha } { 2 } } ( m + \sigma _ { r i } ^ { 2 } r _ { i } ^ { - \alpha } z ) ^ { - m } } { 2 ( r _ { i } ^ { \alpha } m + \sigma _ { r i } ^ { 2 } z ) \Gamma ( m ) } ) \left(2 l_{i}^{\frac{\alpha}{2}}\left(r_{i}^{\alpha} m+\sigma_{r i}^{2} z\right) \Gamma(m)^{2}\right.\right. \\
& \times{ }_{2} F_{1}\left(\frac{1}{2}+m ; \frac{1}{2}+m ;-\frac{1}{2} ;-\frac{z \vartheta_{c}^{2} r_{i}^{-\alpha}}{m^{2} r_{i}^{\alpha}+z m \sigma_{r i}^{2}}\right)-\frac{j r_{i}^{\frac{-\alpha}{2}} \sqrt{m+\sigma_{r i}^{2} r_{i}^{-\alpha} z}}{m^{\frac{5}{2}} \vartheta_{c} \sqrt{z}}\left(\frac{1}{2}+m\right)^{2} \\
& \times\left\{\left(m^{2}\left(l_{i} r_{i}\right)^{\alpha}+z\left(\sigma_{r}^{2} r_{i}^{\alpha} m+\vartheta_{c}^{2}\right)\right)_{2} F_{1}\left(\frac{1}{2}+m ; \frac{1}{2}+m ;-\frac{1}{2} ;-\frac{z \vartheta_{c}^{2} r_{i}^{-\alpha}}{m^{2} r_{i}^{\alpha}+z m \sigma_{r i}^{2}}\right)\right. \\
&\left.\left.\left.\left.-\left(m^{2}\left(l_{i} r_{i}\right)^{\alpha}+z\left(\sigma_{r i}^{2} r_{i}^{\alpha} m+2(1+2 m) \vartheta_{c}^{2}\right)\right)_{2} F_{1}\left(\frac{1}{2}+m ; \frac{1}{2}+m ; \frac{1}{2} ;-\frac{z \vartheta_{c}^{2} r_{i}^{-\alpha}}{m^{2} r_{i}^{\alpha}+z m \sigma_{r i}^{2}}\right)\right\}\right)\right)\right]+R_{C} .
\end{aligned}
$$

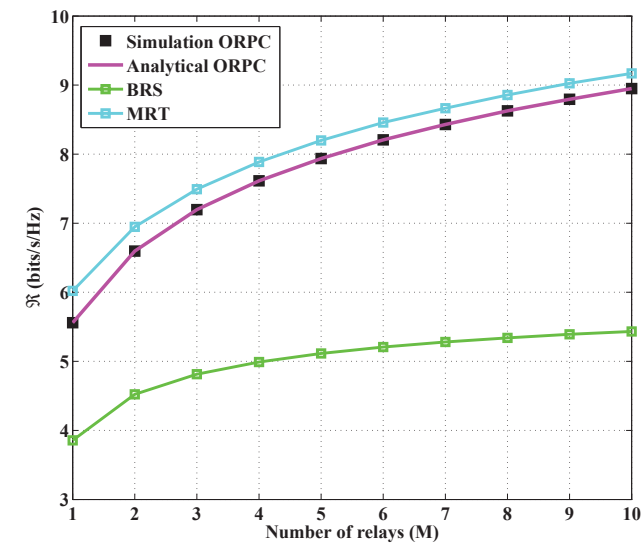

Figure 1: $\mathrm{SE}$ as function of $\mathrm{M}$ in Rayleigh fading channels

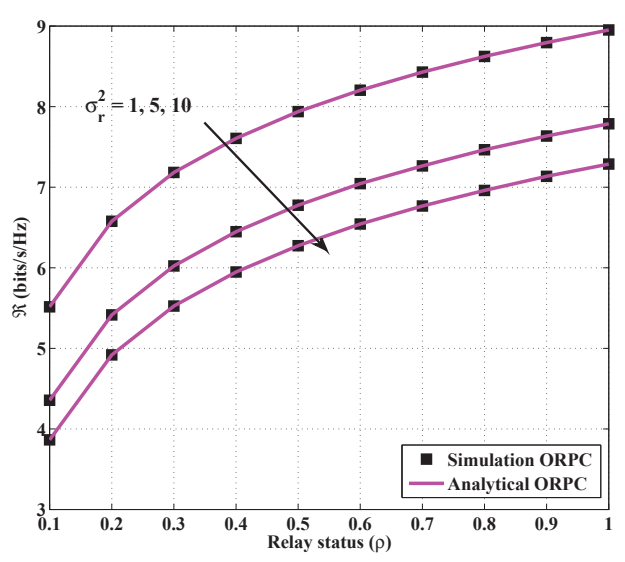

Figure 3: SE as $\rho$ in Rayleigh fading channels

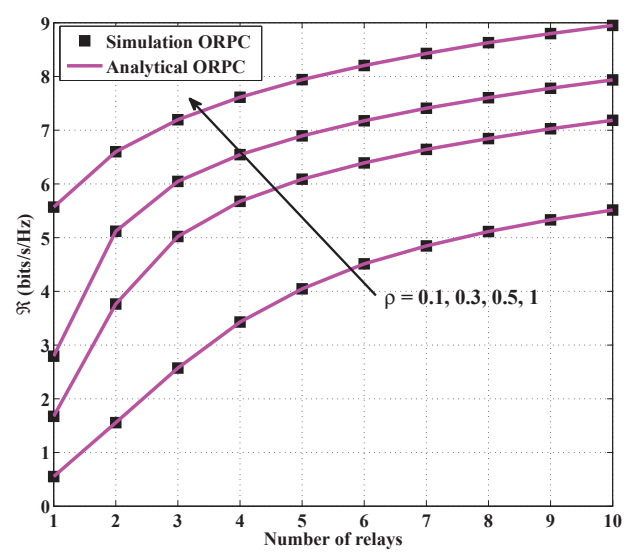

Figure 2: $\mathrm{SE}$ as function of $\mathrm{M}$ in Rayleigh fading channels

In Fig. 3, the SE is plotted as function of the relay status $(\rho)$ for different values of $\sigma_{r}^{2}(1,5,10 \mathrm{watt} / \mathrm{Hz})$ with $\sigma_{d}^{2}=1 \mathrm{watt} / H z$, and $M=10$. It can be seen that as $\rho$ increases, the performance of the system increases. However, as would be expected, increasing noise power of the relays has negative effect on the system performance, for example, increasing $\sigma_{r}^{2}$ from 1 to $10 \mathrm{watt} / \mathrm{Hz}$ gives marked decline in system performance.

\section{B. Nakagami-m Fading Channel}

The results obtained for the case of the Nakagami-m channel show similar trends to those obtained for the Rayleigh fading channel, i.e. the SE increases as the number of cooperating relays increases. Fig. 4, shows SE of the three different schemes as function of the number of cooperating relays, $M$, when $\sigma_{r}^{2}=\sigma_{d}^{2}=1 \mathrm{watt} / \mathrm{Hz}$. Again, the performance of ORPC is significantly better than that of the BRS, but less than the MRT. As expected, increasing the number of cooperating relay nodes $(M)$ positively enhances the SE performance.

Fig. 5, shows system performance as the number of cooperating channels increases, and $\rho$ increase from 0.1 to 1 , with $\sigma_{r}^{2}=\sigma_{d}^{2}=1 \mathrm{watt} / \mathrm{Hz}$. It can be seen that system performance improves - the signal between source and destination is increased - with increase in either $M$ or $\rho$. In Fig. 6 , the SE is plotted as a function of the relay status $(\rho)$ for different values of $\sigma_{r}^{2}(0,1,5,10 \mathrm{watt} / \mathrm{Hz})$ when $\sigma_{d}^{2}=1 \mathrm{watt} / \mathrm{Hz}$ and $M=10$. It can be seen that as $\rho$ increases the performance of the system also increases. As expected, $\sigma_{r}^{2}$ has a negative effect on system performance, as $\sigma_{r}^{2}$ increases from 0 to 10 


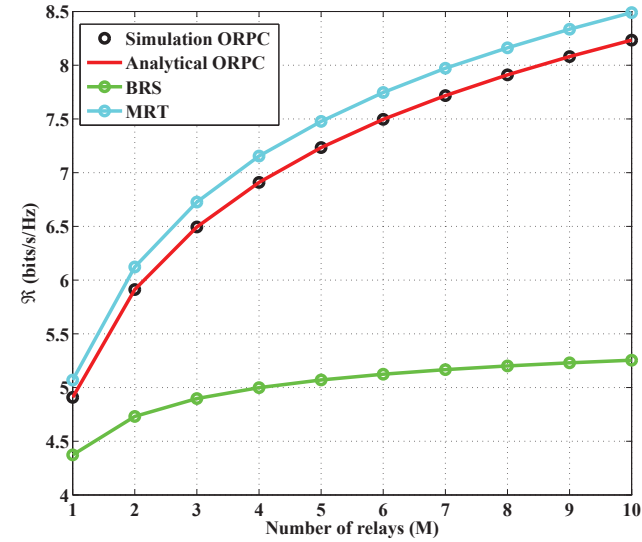

Figure 4: $\mathrm{SE}$ as function of $\mathrm{M}$ in Nakagami-m fading channel

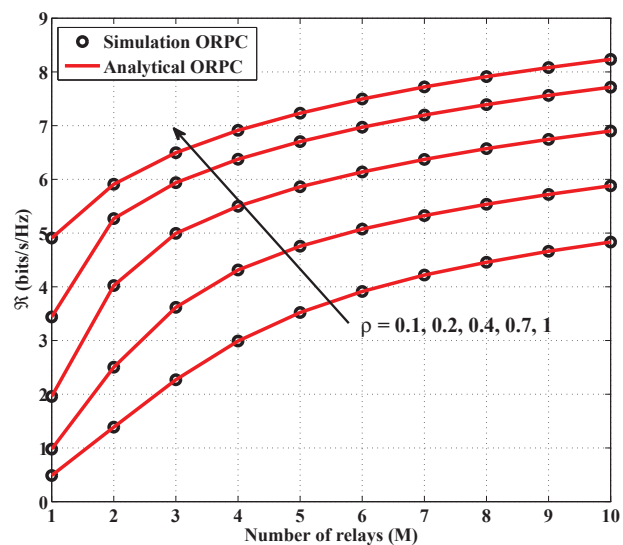

Figure 5: $\mathrm{SE}$ as function of $\mathrm{M}$ in Nakagami-m fading channel

watt/hz, the SE decreases dramatically.

\section{CONCLUSIONS}

In this paper, we analysed in detail the performance of a cooperative diversity network using ORPC scheme, with $M$ cooperating relays used to enhance data transmission when there is no direct link between source and destination. The relays are located between the source and destination, and status of each relay is randomly considered. We derive analytical expressions for the overall SE, and assume that the channels between the source, relays and destinations experience two different fading channels - Rayleigh and Nakagami$\mathrm{m}$. We validated the accuracy of our results with Monte Carlo simulations. We compare the performance of the ORPC model with BRS and MRT and confirmed that the performance of the ORPC cooperative diversity system is significantly better than the BRS, but not as good as the MRT. We also examined the impact of increasing the number of relays on the performance of such a system.

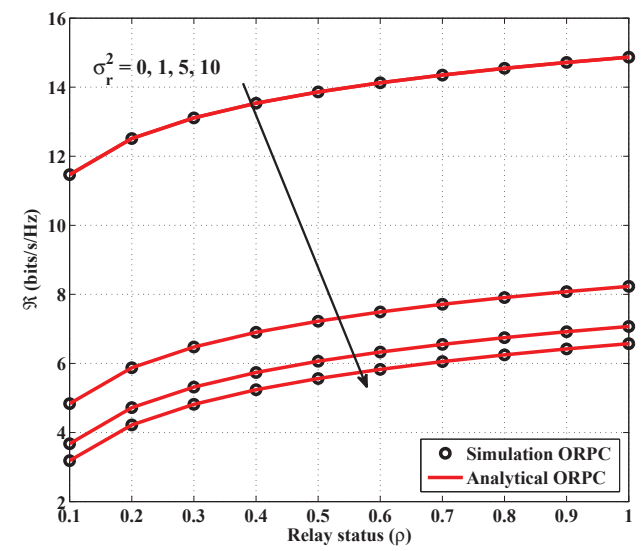

Figure 6: SE as function of $\rho$ in Nakagami-m fading channel

\section{REFERENCES}

[1] X. Zhang, M. O. Hasna, and A. Ghrayeb, "An adaptive transmission scheme for two-way relaying with asymmetric data rates," IEEE Trans. Veh. Technol, vol. 65, no. 3, pp. 1477-1491, 2016.

[2] M. Sami, N. Noordin, and M. Khabazian, "A TDMA-based cooperative MAC protocol for cognitive networks with opportunistic energy harvesting," IEEE Commun. Lett., vol. PP, no. 99, pp. 1-1, 2016.

[3] A. Sendonaris, E. Erkip, and B. Aazhang, "User cooperation diversity. part I. system description,” IEEE Trans. Commun., vol. 51, no. 11, pp. 1927-1938, Nov. 2003.

[4] W. Su, A. Sadek, and K. Liu, "SER performance analysis and optimum power allocation for decode-and-forward cooperation protocol in wireless networks," in IEEE Int. Commun. Conf. (ICC), vol. 2, Mar. 2005, pp. 984-989 Vol. 2.

[5] L. Zheng and D. Tse, "Diversity and multiplexing: a fundamental tradeoff in multiple-antenna channels," IEEE Trans. Inf. Theory, vol. 49, no. 5, pp. 1073-1096, 2003.

[6] H. Fu, M. Crussiere, and M. Helard, "Ber analysis for equal gain transmission in downlink multiuser MIMO systems," IEEE Commun. Lett., vol. 4, no. 5, pp. 533-536, 2015.

[7] M. Maaz, M. Helard, P. Mary, and M. Liu, "Performance analysis of time-reversal based precoding schemes in MISO-OFDM systems," in IEEE Veh. Technol. Conf. (VTC), 2015, pp. 1-6.

[8] M. S. P. Facina, H. A. Latchman, H. V. Poor, and M. V. Ribeiro, "Cooperative in-home power line communication: Analyses based on a measurement campaign," IEEE Trans. Commun., vol. 64, no. 2, pp. 778-789, 2016.

[9] S. Ikki and M. Ahmed, "Performance of cooperative diversity using equal gain combining (EGC) over Nakagami-m fading channels," IEEE Trans. Commun., vol. 8, no. 2, pp. 557-562, Feb. 2009.

[10] Y. Jing and H. Jafarkhani, "Single and multiple relay selection schemes and their achievable diversity orders," IEEE Trans. Commun., vol. 8, no. 3, pp. 1414-1423, Mar. 2009.

[11] M. Hasna and M.-S. Alouini, "End-to-end performance of transmission systems with relays over Rayleigh-fading channels," IEEE Trans. Wireless Commun., vol. 2, no. 6, pp. 1126-1131, Nov. 2003.

[12] P. Anghel and M. Kaveh, "Exact symbol error probability of a cooperative network in a Rayleigh-fading environment," IEEE Trans. Wireless Commun., vol. 3, no. 5, pp. 1416-1421, 2004.

[13] K. Hamdi, "A useful lemma for capacity analysis of fading interference channels," IEEE Trans. Commun., vol. 58, no. 2, pp. 411-416, Feb. 2010.

[14] _ , "Accurate performance analysis of OFDM over Nakagami-m fading with nonuniform phase distribution," in IEEE Global Telecommun. Conf. (GLOBECOM), Dec. 2010, pp. 1-5.

[15] _ _ "Some notes on the fourier transform methods for error-rate analysis of equal-gain combining," IEEE Trans. Commun., vol. 58, no. 12, pp. 3364-3368, Dec. 2010.

[16] M. Abramowitz and I. A. Stegun, Handbook of Mathematical Functions. New York, NY, USA: Dover, 1965. 